Dear Author,

Please, note that changes made to the HTML content will be added to the article before publication, but are not reflected in this PDF.

Note also that this file should not be used for submitting corrections. 


\title{
Evaluating the accuracy of ESR dose determination of pseudo-Early Pleistocene fossil tooth enamel samples using dose recovery tests
}

\author{
Mathieu Duval ${ }^{\mathrm{a}, \mathrm{b}}$, * \\ a Program of Geochronology, Centro Nacional de Investigación Sobre la Evolución Humana (CENIEH), Spain \\ ${ }^{\mathrm{b}}$ Research School of Earth Sciences, The Australian National University, Canberra, Australia
}

\section{H I G H L I G H T S}

- Dose recovery tests performed on fossil tooth enamel with $D_{E}$ values $>1,000 \mathrm{~Gy}$.

- Several fitting functions were used.

- The SSE function does not correctly describe the behavior of ESR signal.

- Using a DSE with data weighed by $1 / \mathrm{I}^{2}$ produce the most accurate $\mathrm{D}_{\mathrm{E}}$ results.

- The SSE might nevertheless produce fairly consistent results under certain conditions.

\section{A R T I C L E I N F O}

\section{Article history:}

Received 13 January 2015

Received in revised form

27 May 2015

Accepted 6 June 2015

Available online $\mathrm{xxx}$

\section{Keywords:}

ESR dating/dosimetry

Fossil tooth enamel

Early Pleistocene

Dose recovery test

\begin{abstract}
A B S T R A C T
In ESR dating of Early Pleistocene fossil tooth enamel samples, the fitting function used for the evaluation of the $D_{E}$ value is undoubtedly among the major sources of uncertainty. Dose recovery tests performed on fossil tooth enamel showing $D_{\mathrm{E}}$ values $>1,000 \mathrm{~Gy}$ demonstrate: (i) that high precision ESR measurements $(<0.5 \%)$ and high $\mathrm{D}_{\mathrm{E}}$ reproducibility $(<5 \%)$ may be achieved; (ii) the appropriateness of the Double Saturating Exponential (DSE) fitting function for ESR dose reconstruction. In contrast, the SSE function, which has been almost exclusively used so far, does simply not correctly describe the behavior of the radiation induced ESR signal of tooth enamel with the dose.

Several fitting functions and data weighting options were tested and the combination of a DSE with data weighed by the inverse of the square intensities is the procedure providing the most accurate $D_{\mathrm{E}}$ results. However, the SSE may nevertheless sometimes produce consistent results if $D_{\max }$ does not exceed $6^{*} \mathrm{D}_{\mathrm{E}}$. Further work is required in that direction in order to determine more precisely in which conditions the SSE could be used as a fair approximation of the DSE function for these samples.
\end{abstract}

() 2015 Published by Elsevier Ltd.

\section{Introduction}

Over the last decade, an extensive work has been dedicated to the evaluation of the exact potential and the current limitations of the ESR dating method specifically applied to Early Pleistocene ( $>0.78 \mathrm{Ma}$ ) fossil tooth enamel samples, with the idea to reach a better understanding of the method and identify the main sources of uncertainty involved in the dating process (e.g. Duval et al., 2009, 2011a and b, 2012a and b, 2013, in press). The long term objective of this ongoing investigation is to improve the reliability and accuracy

* Program of Geochronology, Centro Nacional de Investigación sobre la Evolución Q1 Humana (CENIEH), Spain.

E-mail address: mathieu.duval@cenieh.es. of this dating method, which is a major challenge in archaeology given that ESR is one of the very few numerical methods that can be used to date the earliest hominin occupations in non-volcanic context in Europe (e.g. Duval et al., 2012), Africa (e.g. Curnoe et al., 2001; Schwarcz et al., 1994) and Asia (e.g. Shao et al., 2014; Han et al., 2012; Tiemei et al., 2001).

A synthesis based on 20 Early Pleistocene teeth from various Spanish localities recently published by Duval et al. (2012) showed that these "old" samples are usually mainly characterized by: high ${ }^{230} \mathrm{Th} /{ }^{234} \mathrm{U}$ activity ratios that may sometimes exceed secular equilibrium, high Uranium concentrations (several tens of ppm or more in dentine) and high equivalent dose $\left(D_{E}\right)$ values that are in most cases $>1000 \mathrm{~Gy}$. In particular, the magnitude of these $\mathrm{D}_{\mathrm{E}}$ values raised many new questions about the reliability of the ESR dose reconstruction procedure that has been routinely used so far, 
in particular regarding the selection of an appropriate fitting function (e.g. Duval et al., 2009).

Since the first ESR dating applications to fossil tooth enamel in the mid 1980s' (e.g. Grün et al., 1985), the use of a linear fitting function has been rapidly abandoned (Grün et al., 1989; Grün, 1996) and replaced by a single saturating exponential function (SSE) in order to take into consideration the saturation of the ESR signal that occur at high irradiation doses. From a physical perspective, this saturation may be explained by the presence of a finite number of precursors available in a given sample and thus a decreasing probability to create radicals with the increasing irradiation dose (Apers et al., 1981). The use of this SSE was also a way to indirectly assume that the radiation induced signal was dominated by a single component, which was found later to be incorrect (e.g. Vanhaelewyn et al., 2000). So far, the SSE function has been exclusively used for ESR dose reconstruction of fossil tooth enamel during several decades, but some limitations have been progressively observed, in particular in the case of samples with high equivalent dose $\left(D_{E}\right)$ values (Chen, 1997). In that regard, Duval et al. (2009) highlighted some clear issues with the use of the SSE in the specific case of Early Pleistocene teeth. For example, these authors showed the strong impact of the maximum irradiation dose $\left(D_{\max }\right)$ on the $D_{E}$ value, which was constantly increasing with the successive addition of ESR points at high doses. This systematic trend demonstrated the impossibility for this function to describe the behavior of the ESR signal at high irradiation doses. To address this issue, they proposed the use of a Double Saturating Exponential function (DSE) instead, which was found not only to be more appropriate for the experimental data, but also more in agreement with the recent advances on the understanding of the ESR signal of fossil tooth enamel and the identification of several types of radiation-induced $\mathrm{CO}_{2}^{-}$radicals contributing to the main signal (Joannes-Boyau and Grün, 2011 and references therein). More recently, Duval et al. (2013) completed the study by proposing the combination of two types of DSE functions in order to fully encompass the uncertainty associated to the variability of the relative proportions of the various types of $\mathrm{CO}_{2}^{-}$among the samples, and by defining some empirical criteria to ensure a reliable fitting.

In ESR dose reconstruction of fossil tooth enamel, the equivalent dose $\left(D_{E}\right)$ is systematically obtained via the additive dose method, i.e. through a back extrapolation of the fitting function to the X-axis. Consequently, and in contrast with the regenerative dose method, the $D_{E}$ value is thus very dependent on the choice of the fitting function. For example, Duval et al. (2009) showed that the relative deviation between the $D_{E}$ values obtained from different functions is significantly increasing along with the magnitude of the $D_{E}$ value. In the case of samples showing $D_{E}$ values $>1000 \mathrm{~Gy}$, the systematic deviation between the $D_{E}$ results derived either from a SSE or a DSE function was estimated to be around $23 \%$ in average, while it was $<10 \%$ for $D_{E}$ values $<300 \mathrm{~Gy}$. In other words, the further goes the back extrapolation (= the larger is the $\mathrm{D}_{\mathrm{E}}$ ), the higher is the influence of the fitting function on the final $D_{E}$ value. Consequently, if the fitting function must be considered as a significant source of uncertainty in ESR dating of fossil tooth enamel in general, its importance is even greater in the specific case of Early Pleistocene samples.

However, despite a series of evidence showing the appropriateness of the DSE and the major limitations of the SSE (e.g. Duval et al., 2009, 2013), there is still some uncertainty about the ability of the DSE function to yield correct $D_{\mathrm{E}}$ values. Actually, the only way to check whether the DSE does not systematically provide overestimated or underestimated results would be to work with known-dose samples. This may be done via "dose recovery" tests that are usually designed to assess the appropriateness of a standard analytical procedure by evaluating whether a laboratory given dose may be experimentally recovered with accuracy. This test is easily performed when the signal may be zeroed without modifying the structure and chemical composition of the sample, like in ESR and OSL dating of optically bleached quartz and feldspar grains (e.g. Asagoe et al., 2011; Murray and Wintle, 2003). However, this is not the case for tooth enamel, since the ESR signal cannot be fully reset by sunlight or by heat. UV irradiations actually create a radiation-induced signal (e.g. Nilsson et al., 2001), while thermal Q2 annealing at increasing temperature induces a decrease of the ESR intensity but is accompanied with some irreversible major changes in the crystalline structure of tooth enamel (e.g. Brik et al., 1996, and 1997), making thus impossible the use of these procedures for dose recovery tests. Consequently, the best strategy for working with known-dose samples consists in selecting tooth samples that have accumulated a very small dose, so that it could be considered as negligible in comparison with a geological dose accumulated over a hundreds of ka. In that regard, recent fossil teeth should be preferred in first instance to modern enamel samples given the existing differences in structure and composition (e.g. Kohn et al., 1999) that may induce specific behaviors with the irradiation dose, even though it is worth mentioning that the very few comparison studies published on this aspect did not lead to identify significant differences in terms of radiation sensitivities (Rink and Schwarcz, 1994, 1995).

Consequently, three recent fossil teeth were selected for the present study and gamma irradiated at $\sim 1500 \mathrm{~Gy}$, in order to simulate a $D_{E}$ value that may be commonly found in Early Pleistocene tooth samples (e.g. Duval et al., 2013). Samples were then analyzed following a Multiple Aliquot Additive (MAA) dose approach, the standard procedure in ESR dating of fossil teeth (e.g. Duval et al., 2011b, 2012b) and several fitting functions were finally used to derive $D_{E}$ values in order to see whether the laboratory given dose could be accurately recovered. The deviations observed between the calculated $D_{E}$ value and the expected $D_{E}$ are presented and discussed in this work.

\section{Material and methods}

Three teeth (MOD1301, MOD1302 and MOD1303) were collected from a protohistoric site in France and prepared following a standard ESR dating procedure. The enamel layer of the vestibular side was extracted and then cleaned using a dentist drill. Clean enamel fragments were then ground and sieved to obtain a powder of $100-200 \mu \mathrm{m}$.

The first step of the analytical procedure was to make sure that the natural dose registered over time by the samples would not significantly interfere with the laboratory given dose. To do so, a first set of four powder aliquots were selected from each sample and three of them were gamma irradiated at 10, 50 and $100 \mathrm{~Gy}$. In this work, all irradiations were performed with a calibrated ${ }^{137} \mathrm{Cs}$ gamma source (dose rate $=7.3 \mathrm{~Gy} / \mathrm{min}$ ). Then, for each sample the gamma irradiated aliquots were measured by ESR together with the natural one (see details about the experimental conditions later). ESR spectra and Dose response curves (DRCs) are shown in Fig. 1. For two samples (MOD1301 and MOD1303), the natural dose was estimated to be around $1.5 \mathrm{~Gy}$, while it was virtually null for MOD1302. These values can thus be considered as negligible in comparison with the laboratory dose that will be given in the next step of the procedure.

Then, each natural enamel sample was split into 16 aliquots. First, all aliquots of a given tooth were irradiated together and given a pre-dose of $1491 \mathrm{~Gy}$ in order to simulate a standard $\mathrm{D}_{\mathrm{E}}$ value that may be frequently found in Early Pleistocene samples (Duval et al., 2013). Each aliquot was measured by ESR in order to control the homogeneity of the irradiation: results showed that the ESR 

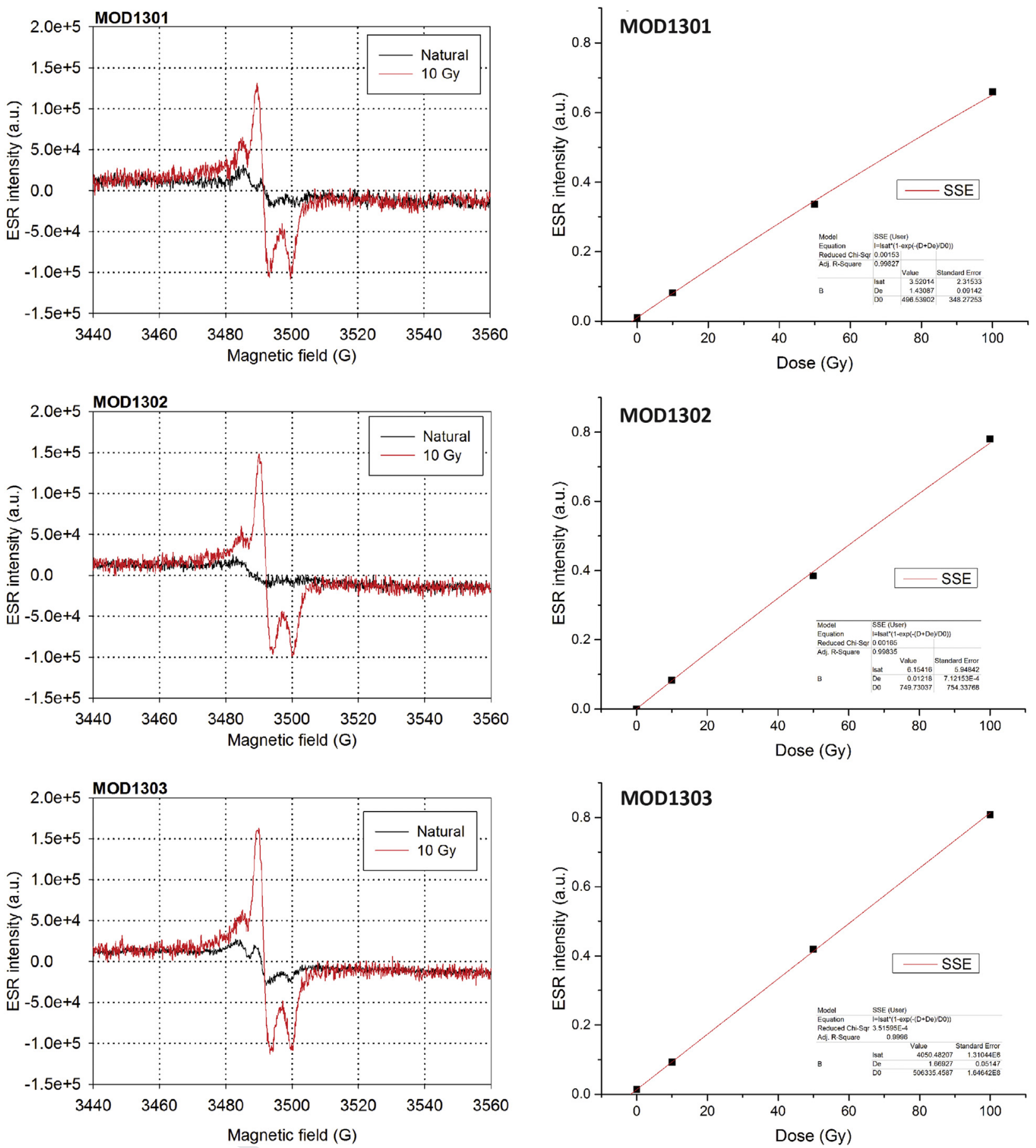

Fig. 1. Estimating the natural dose absorbed by the three teeth. Left column: examples of ESR spectra of the natural and gamma irradiated (10 Gy) aliquots measured for the three samples. Spectra are normalized to 1 scan. ESR measurements were performed at room temperature with the following acquisition parameters: 1 mW microwave power, 1024 points resolution, $13 \mathrm{mT}$ sweep width, $100 \mathrm{kHz}$ modulation frequency, $0.1 \mathrm{mT}$ modulation amplitude, $40 \mathrm{~ms}$ conversion time and $10 \mathrm{~ms}$ time constant. Right column: dose response curves obtained for the three samples. The estimation of the natural dose was derived from the fitting of a SSE function through the experimental data points (weighting by $1 / \mathrm{I}^{2}$ ). Sample MOD01 and MOD02 show an apparent $\mathrm{D}_{\mathrm{E}}$ value around $1.5 \mathrm{~Gy}$, while the value obtained for MOD02 is virtually null.

intensities of all aliquots vary within narrow range, between 1.0 and $1.2 \%$ ( 1 relative standard deviation) (see supplementary information, Fig. S1). This variability is similar to the experimental uncertainty that is usually observed for the ESR measurements of tooth enamel (Duval et al., 2013), suggesting that the uncertainty associated to a potential heterogeneous irradiation of a given batch of aliquots may be considered as negligible. These results indicate that all aliquots effectively received similar doses. This variability may be used as a reasonable proxy to estimate the uncertainty on the given dose, i.e. $1491 \pm 18.0,1491 \pm 17.9$ and $1491 \pm 17.0 \mathrm{~Gy}$ for samples MOD1301, MOD1302 and MOD1303, respectively. About 7 months later, a second round of gamma irradiations was organized in order to build a standard DRC and to check whether the given dose can be recovered. All but one aliquots were irradiated at 
increasing dose, using a sub-exponential dose step distribution (Grün and Rhodes, 1992), as follows: 99, 248, 495, 792, 1284, 2480, 3962, 5943, 8915, 12,877, 17,829, 24,756, 34,661, 44,566 and $59,561 \mathrm{~Gy}$. The remaining aliquot kept unirradiated during this second round of irradiation was thus considered later on as the "natural" one.

ESR measurements were carried out at the CENIEH (Burgos, Spain), with a EMXmicro 6/1 Bruker ESR spectrometer coupled to a standard rectangular ER 4102ST cavity, several months after the irradiation in order to let the radiation induced temporary signals decay (Hoffmann and Mangini, 2002). To ensure constant experimental conditions over time, the temperature of the water circulating in the magnet is controlled and stabilized at $18^{\circ} \mathrm{C}$ by a watercooled Thermo Scientific NESLAB ThermoFlex 3500 chiller, and the temperature of the room is kept constant at $20^{\circ} \mathrm{C}$ by an air conditioning unit. ESR measurements were performed at room temperature with the following acquisition parameters: 1-5 scans, $1 \mathrm{~mW}$ microwave power, 1024 points resolution, $15 \mathrm{mT}$ sweep width, $100 \mathrm{kHz}$ modulation frequency, $0.1 \mathrm{mT}$ modulation amplitude, 20 ms conversion time and $5 \mathrm{~ms}$ time constant. For a given sample, the receiver gain value was optimized according to the ESR signal of the most irradiated aliquot and the same value was then used for all the aliquots from a given sample. In order to ensure similar resonance conditions in the ESR cavity for all the aliquots of a given sample, each aliquot was carefully weighted in the ESR tube. For the three samples, the mass per aliquot was $\sim 70 \mathrm{mg}$, and a maximum variation of $1 \mathrm{mg}$ in the mass of all aliquots from a given tooth sample was tolerated, corresponding to a relative variability $<1.4 \%$ in weight for each tooth sample. This results in a variation of the enamel powder height in the tube of $<0.5 \mathrm{~mm}$ between aliquots. Measurements were performed using a pedestal inserted in the cavity, so that the uncertainty associated to the variation of the vertical position of the tube from one aliquot to another was minimized. Each aliquot of a given sample was measured three times after $\sim 120^{\circ}$ rotation in the cavity in order to consider the uncertainty on the angular dependence of the signal. This uncertainty is classically lower than $1 \%$. Basically, all aliquots of a given sample were successively measured in a short time $(<1 \mathrm{~h})$. This procedure was repeated over three distinct days without removing the enamel powder from the ESR tubes between measurements. The ESR intensities were extracted from peak-to-peak measurements amplitudes of the ESR signal of enamel (T1-B2, see Grün et al., 1998), and then corrected by the corresponding receiver gain, number of scans and aliquot mass.

Fitting procedures were carried out with the Microcal OriginPro 9.1 software using a Levenberg-Marquardt algorithm by chi-square minimization. Further details about the potential of this algorithm for non linear fitting may be found in Hayes et al. (1998). Data were either non weighted or weighted by the inverse of the squared ESR intensity (1/12) (Grün and Brumby, 1994; Grün and Rhodes, 1992). Four fitting functions were used for dose assessment, following the equations shown in Table 1: a double saturating exponential (DSE) function, a modified version of this DSE (DSE2), an exponential plus linear function (EXPLIN) and a single saturating exponential (SSE) function. Further details about these functions may be found in Duval et al. (2009, 2013).

In the following sections, $\mathrm{D}_{\mathrm{E}}$ calculations were successively carried out for each sample: (i) based on the ESR intensities measured for each independent measurement (Table 2), (ii) by averaging for a given aliquot the ESR intensities derived from the three repeated measurements (Table 3), (iii) by pooling all the ESR intensities (= all the measurements performed at a given dose are put together for the fitting procedure, see details in Duval et al., 2013) derived from the three repeated measurements (Table 4).

\section{Results and discussion}

\subsection{ESR measurement repeatability}

Each sample was measured three times on different days in order to evaluate the repeatability of the ESR measurements. From one measurement to the other, the ESR intensities of each aliquot showed a very small variation of $0.5 \%, 0.4 \%$ and $0.3 \%$ on average ( 1 standard deviation) for samples MOD1301, MOD1302 and MOD1303, respectively. For a given aliquot, variations were systematically $<0.9 \%$, except for one aliquot $(1.7 \%)$, demonstrating that ESR measurements were highly repeatable.

The $D_{E}$ value was calculated by fitting the various functions for each repeated measurement (Table 2). Each dose response curve (DRC) shows an excellent goodness-of-fit (adjusted $r^{2}>0.99$ ), whatever the function used. All the $D_{E}$ values are highly consistent for a given function: they show very small variations $(<5 \%)$ over the repeated measurements. In particular, the results derived from the use of the SSE show systematically less scatter, mainly because this is the function with the lowest number of fitted parameters (3). In contrast, the highest variability is systematically obtained with the DSE function using equal weights, but it does not exceed $4.3 \%$.

$D_{E}$ values derived from the DSE function (data weighting by $1 / \mathrm{I}^{2}$ ) show a variability of $1.5 \%, 0.75 \%$ and $2.0 \%$ for samples MOD01, MOD02 and MOD03, respectively. These values are significantly lower to those observed by Duval et al. (2013) on other Early Pleistocene samples (between $4.1 \%$ and $7.8 \%$ ). Similarly, in that study the variability of the $D_{\mathrm{E}}$ obtained from the SSE function was ranging from $0.4 \%$ to $2.9 \%$ depending on the sample $(n=11)$, whereas in the present work it is between 0.2 and $1.1 \%(n=3)$. This improved reproducibility may be mostly explained by the combination of two factors: (i) the use of a pedestal inserted in the cavity that minimized the uncertainty associated to slight variations of the vertical position of the tubes, and (ii) the higher number of data points in the DRCs.

These data show that it is possible to achieve an excellent measurement precision and $\mathrm{D}_{\mathrm{E}}$ reproducibility in ESR dosimetry of fossil tooth enamel, even with samples showing a $D_{E}>1000 \mathrm{~Gy}$. The correctness of the $D_{E}$ values is evaluated in the next section.

\subsection{Dose recovery tests}

For each sample, mean ESR intensities were derived from the

Table 1

Equations of the various fitting functions used in the present work.

\begin{tabular}{|c|c|c|}
\hline Function & Equation & Fitted param. \\
\hline $\begin{array}{l}\text { DSE } \\
\text { DSE2 } \\
\text { EXPLIN } \\
\text { SSE }\end{array}$ & $\begin{array}{l}I=I_{1} *\left(1-e^{\left.-\left(D+D_{E}\right) / D_{1}\right)}\right)+I_{2} *\left(1-e^{\left.-\left(D+D_{E}\right) / D_{2}\right)}\right) \\
I_{1} *\left(1-e^{\left.-\left(D+D_{E}\right) / D_{1}\right)}\right)+I_{2} *\left(1-e^{\left.-(D) / D_{2}\right)}\right) \\
I_{1} *\left(1-e^{\left.-\left(D+D_{E}\right) / D_{1}\right)}\right)+I_{2} * D \\
I=I_{\text {Sat }} *\left(1-e^{\left.-\left(D+D_{E}\right) / D_{0}\right)}\right)\end{array}$ & $\begin{array}{l}\text { (5): } I_{1}, I_{2}, D_{1}, D_{2}, D_{E} . \\
(4): I_{1}, I_{2}, D_{1}, D_{2}, D_{E} . \\
(4): I_{1}, I_{2}, D_{1}, D_{E} \\
\text { (3): } I_{\text {sat }}, D_{0}, D_{E} .\end{array}$ \\
\hline
\end{tabular}


Table 2

Reproducibility of the $\mathrm{D}_{\mathrm{E}}$ values calculated with each fitting function. Each sample was measured three times.

\begin{tabular}{|c|c|c|c|c|c|c|c|c|c|c|c|c|c|}
\hline \multirow[t]{2}{*}{ Function } & \multirow{2}{*}{$\begin{array}{l}\text { Meas. } \\
\text { number }\end{array}$} & \multicolumn{4}{|l|}{ MOD1301 } & \multicolumn{4}{|l|}{ MOD1302 } & \multicolumn{4}{|l|}{ MOD1303 } \\
\hline & & $\mathrm{D}_{\mathrm{E}}(\mathrm{Gy})$ & Adj. $\mathrm{r}^{2}$ & Mean $D_{E}$ & $\begin{array}{l}\text { Relative } \\
\text { st. dev. }\end{array}$ & $\mathrm{D}_{\mathrm{E}}(\mathrm{Gy})$ & Adj. $r^{2}$ & Mean $\mathrm{D}_{\mathrm{E}}$ & $\begin{array}{l}\text { Relative } \\
\text { st. dev. }\end{array}$ & $\mathrm{D}_{\mathrm{E}}(\mathrm{Gy})$ & Adj. $r^{2}$ & Mean $D_{E}$ & $\begin{array}{l}\text { Relative } \\
\text { st. dev. }\end{array}$ \\
\hline \multirow[t]{3}{*}{$\operatorname{DSE}\left(\mathrm{W} \sim 1 / \mathrm{I}^{2}\right)$} & $\# 1$ & $1488 \pm 140$ & 0.9977 & \multirow[t]{3}{*}{1465} & \multirow[t]{3}{*}{$1.5 \%$} & $1550 \pm 54$ & 0.9996 & \multirow[t]{3}{*}{1562} & \multirow[t]{3}{*}{$0.75 \%$} & $1480 \pm 70$ & 0.9995 & \multirow[t]{3}{*}{1515} & \multirow[t]{3}{*}{$1.9 \%$} \\
\hline & \#2 & $1459 \pm 119$ & 0.9985 & & & $1573 \pm 52$ & 0.9996 & & & $1535 \pm 62$ & 0.9996 & & \\
\hline & \#3 & $1447 \pm 141$ & 0.9982 & & & $1564 \pm 53$ & 0.9996 & & & $1529 \pm 65$ & 0.9996 & & \\
\hline \multirow[t]{3}{*}{ DSE (EW) } & \#1 & $1625 \pm 129$ & 0.9992 & \multirow[t]{3}{*}{1596} & \multirow[t]{3}{*}{$4.3 \%$} & $1573 \pm 69$ & 0.9997 & \multirow[t]{3}{*}{1601} & \multirow[t]{3}{*}{$1.5 \%$} & $1678 \pm 92$ & 0.9996 & \multirow[t]{3}{*}{1719} & \multirow[t]{3}{*}{$2.1 \%$} \\
\hline & \#2 & $1518 \pm 132$ & 0.9994 & & & $1620 \pm 70$ & 0.9997 & & & $1746 \pm 89$ & 0.9995 & & \\
\hline & \#3 & $1645 \pm 142$ & 0.9992 & & & $1610 \pm 75$ & 0.9997 & & & $1734 \pm 88$ & 0.9996 & & \\
\hline \multirow[t]{3}{*}{$\operatorname{DSE} 2\left(\mathrm{~W} \sim 1 / \mathrm{I}^{2}\right)$} & $\# 1$ & $1966 \pm 290$ & 0.9977 & \multirow[t]{3}{*}{2003} & \multirow[t]{3}{*}{$2.5 \%$} & $1795 \pm 74$ & 0.9996 & \multirow[t]{3}{*}{1803} & \multirow[t]{3}{*}{$0.4 \%$} & $2004 \pm 123$ & 0.9995 & \multirow[t]{3}{*}{1988} & \multirow[t]{3}{*}{$0.7 \%$} \\
\hline & \#2 & $1982 \pm 230$ & 0.9985 & & & $1806 \pm 73$ & 0.9996 & & & $1982 \pm 125$ & 0.9996 & & \\
\hline & \#3 & $2060 \pm 247$ & 0.9982 & & & $1808 \pm 76$ & 0.9996 & & & $1978 \pm 127$ & 0.9996 & & \\
\hline \multirow[t]{3}{*}{$\operatorname{SSE}\left(\mathrm{W} \sim 1 / \mathrm{I}^{2}\right)$} & $\# 1$ & $1874+109$ & 0.9949 & \multirow[t]{3}{*}{1882} & \multirow[t]{3}{*}{$1.1 \%$} & $1921 \pm 105$ & 0.9957 & \multirow[t]{3}{*}{1926} & \multirow[t]{3}{*}{$0.2 \%$} & $1916 \pm 94$ & 0.9964 & \multirow[t]{3}{*}{1917} & \multirow[t]{3}{*}{$0.1 \%$} \\
\hline & \#2 & $1868 \pm 103$ & 0.9954 & & & $1929+102$ & 0.9960 & & & $1917 \pm 90$ & 0.9968 & & \\
\hline & \#3 & $1905+106$ & 0.9953 & & & $1928 \pm 103$ & 0.9959 & & & $1918 \pm 92$ & 0.9966 & & \\
\hline \multirow{3}{*}{$\begin{array}{l}\text { EXPLIN } \\
\qquad\left(\mathrm{W} \sim 1 / \mathrm{I}^{2}\right)\end{array}$} & $\# 1$ & $1715 \pm 89$ & 0.9975 & 1723 & $1.1 \%$ & $1716 \pm 49$ & 0.9993 & 1725 & $0.4 \%$ & $1747 \pm 56$ & 0.9991 & 1750 & $0.2 \%$ \\
\hline & \#2 & $1709 \pm 80$ & 0.9979 & & & $1730 \pm 46$ & 0.9994 & & & $1753 \pm 48$ & 0.9993 & & \\
\hline & \#3 & $1745 \pm 85$ & 0.9978 & & & $1728 \pm 47$ & 0.9994 & & & $1752 \pm 50$ & 0.9993 & & \\
\hline
\end{tabular}

Table 3

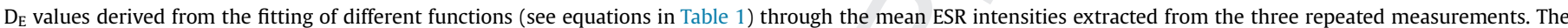

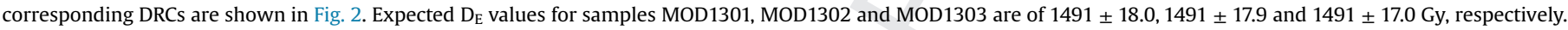

\begin{tabular}{|c|c|c|c|c|c|c|c|c|c|c|}
\hline & \multicolumn{3}{|l|}{ MOD1301 } & \multicolumn{3}{|l|}{ MOD1302 } & \multicolumn{3}{|l|}{ MOD1303 } & \multirow{2}{*}{$\frac{\text { Mean }}{D_{E} / \text { true } D_{E}}$} \\
\hline & $\mathrm{D}_{\mathrm{E}}(\mathrm{Gy})$ & Adj. $r^{2}$ & $\mathrm{D}_{\mathrm{E}} /$ true $\mathrm{D}_{\mathrm{E}}$ & $\mathrm{D}_{\mathrm{E}}(\mathrm{Gy})$ & Adj. $r^{2}$ & $\mathrm{D}_{\mathrm{E}} /$ true $\mathrm{D}_{\mathrm{E}}$ & $\mathrm{D}_{\mathrm{E}}(\mathrm{Gy})$ & Adj. $r^{2}$ & $\mathrm{D}_{\mathrm{E}} /$ true $\mathrm{D}_{\mathrm{E}}$ & \\
\hline $\operatorname{DSE}\left(\mathrm{W} \sim 1 / \mathrm{I}^{2}\right)$ & $1468 \pm 130$ & 0.99824 & 0.98 & $1562 \pm 52$ & 0.99961 & 1.05 & $1514 \pm 65$ & 0.99958 & 1.02 & 1.02 \\
\hline DSE (EW) & $1595 \pm 129$ & 0.99931 & 1.07 & $1600 \pm 70$ & 0.99972 & 1.07 & $1720 \pm 88$ & 0.99958 & 1.15 & 1.10 \\
\hline $\operatorname{DSE} 2\left(\mathrm{~W} \sim 1 / \mathrm{I}^{2}\right)$ & $2006 \pm 251$ & 0.99824 & 1.35 & $1803 \pm 74$ & 0.99961 & 1.21 & $1993 \pm 124$ & 0.99958 & 1.34 & 1.30 \\
\hline $\operatorname{SSE}\left(\mathrm{W} \sim 1 / \mathrm{I}^{2}\right)$ & $1882+105$ & 0.99528 & 1.26 & $1925 \pm 103$ & 0.99584 & 1.29 & $1917 \pm 92$ & 0.99663 & 1.29 & 1.28 \\
\hline $\operatorname{EXPLIN}\left(\mathrm{W} \sim 1 / \mathrm{I}^{2}\right)$ & $1723 \pm 83$ & 0.99783 & 1.16 & $1725 \pm 47$ & 0.99935 & 1.16 & $1750 \pm 51$ & 0.99922 & 1.17 & 1.16 \\
\hline
\end{tabular}

three repeated ESR measurements and then final $D_{E}$ values were calculated for each fitting function presented in Table 1. Numerical results and DRCs are shown in Table 3 and Fig. 2, respectively. If all the functions show an excellent goodness-of-fit (adjusted $r^{2}$ values $>0.99$ ), the SSE systematically shows the lowest value, which means this is the one that least describes the behavior of the ESR signal with the dose.

The results obtained from the three samples show that all the $D_{E}$ values are systematically overestimating the true $D_{\mathrm{E}}$ except in one case (sample MOD1301 with DSE function and weighting by $1 / \mathrm{I}^{2}$ ). However, this overestimation is more or less significant depending on the function. For example, the use of the DSE but without weighting the data provides an overestimation by about $10 \%$ on average of the $D_{E}$. One of the samples has a $D_{E}$ in agreement with the expected value at 1 sigma (MOD1301) and another at $2 \sigma$ (MOD1302). The other functions such as EXPLIN, DSE2 and SSE provide $D_{E}$ values that are systematically (and significantly) overestimated by $16 \%, 28 \%$ and $30 \%$ on average, respectively, as shown in Table 3. These results demonstrate that the SSE does simply not describe the behavior of the radiation induced ESR of tooth enamel with the irradiation dose and therefore cannot provide reliable $\mathrm{D}_{\mathrm{E}}$ results for this data set. In addition, the two other functions, EXPLIN and DSE2, cannot be considered as reliable alternative.

Table 4

DE values obtained from the fitting of a DSE function through the whole experimental data set made by all ESR intensities obtained from the three repeated ESR measurements ( 16 points $* 3=48$ points for each DRC).

\begin{tabular}{llll}
\hline Sample & $\mathrm{D}_{\mathrm{E}}(\mathrm{Gy})$ & Relative error & Adj. $^{2}$ \\
\hline MOD1301 & $1467 \pm 68$ & $4.6 \%$ & 0.99849 \\
MOD1302 & $1562 \pm 28$ & $1.8 \%$ & 0.99964 \\
MOD1303 & $1514 \pm 34$ & $2.2 \%$ & 0.99964 \\
\hline
\end{tabular}

In contrast, the results obtained from the three samples show that on average the DSE (weighting by $1 / \mathrm{I}^{2}$ ) provides a $\mathrm{D}_{\mathrm{E}}$ values that is only $2 \%$ higher than the expected $D_{E}$ value. All samples show a $D_{E}$ result in agreement at 1 sigma with the expected $D_{E}$ value (see also Fig. 3), demonstrating thus the appropriateness of this function to describe the behavior of the ESR signal of tooth enamel with the irradiation dose. However, it is also worth mentioning that the sample MOD1302 showing the best goodness-of-fit and the most precise $D_{E}$ values ( $D_{E}$ errors $<4 \%$ ) is actually the one providing the highest deviation with the expected value $(\sim 5 \%)$. This observation indicates that high precision data does not necessarily ensure accurate results. This issue will be further explored in section 2.4. Relative $D_{E}$ errors are around 3-4\% for samples MOD1302 and MOD1303, while it is larger for MOD1301 ( 9\%). The magnitude of the latter is simply explained by a slightly more scattered DRC, in particular in the low dose region (see Fig. 2 and Adj. $\mathrm{r}^{2}$ value in Table 3 ) inducing thus more uncertainty in the fitting. In addition, one may observe that $D_{E}$ errors are on average higher without weighting than with by $\mathrm{w} \sim 1 / \mathrm{I}^{2}$ ( $5.9 \%$ vs $5.5 \%$ on average). These results are in agreement with those previously published by Grün and Brumby (1994) and Grün (1998), and demonstrate the need to carry out a fitting with data that are weighted by the inverse of the squared intensities in order to reduce both systematic and random errors on the $D_{E}$ value.

\subsection{Influence of $D_{\max }$ on the $D_{E}$ value}

Another criterion to evaluate the appropriateness of a function to fit the experimental data set is to evaluate the influence of the maximum irradiation dose $\left(D_{\max }\right)$ on the $D_{E}$ (see Duval et al., 2009, 2013). By definition, if both parameters are strongly correlated, it may indicate that the function is simply not adapted to the experimental data set. 

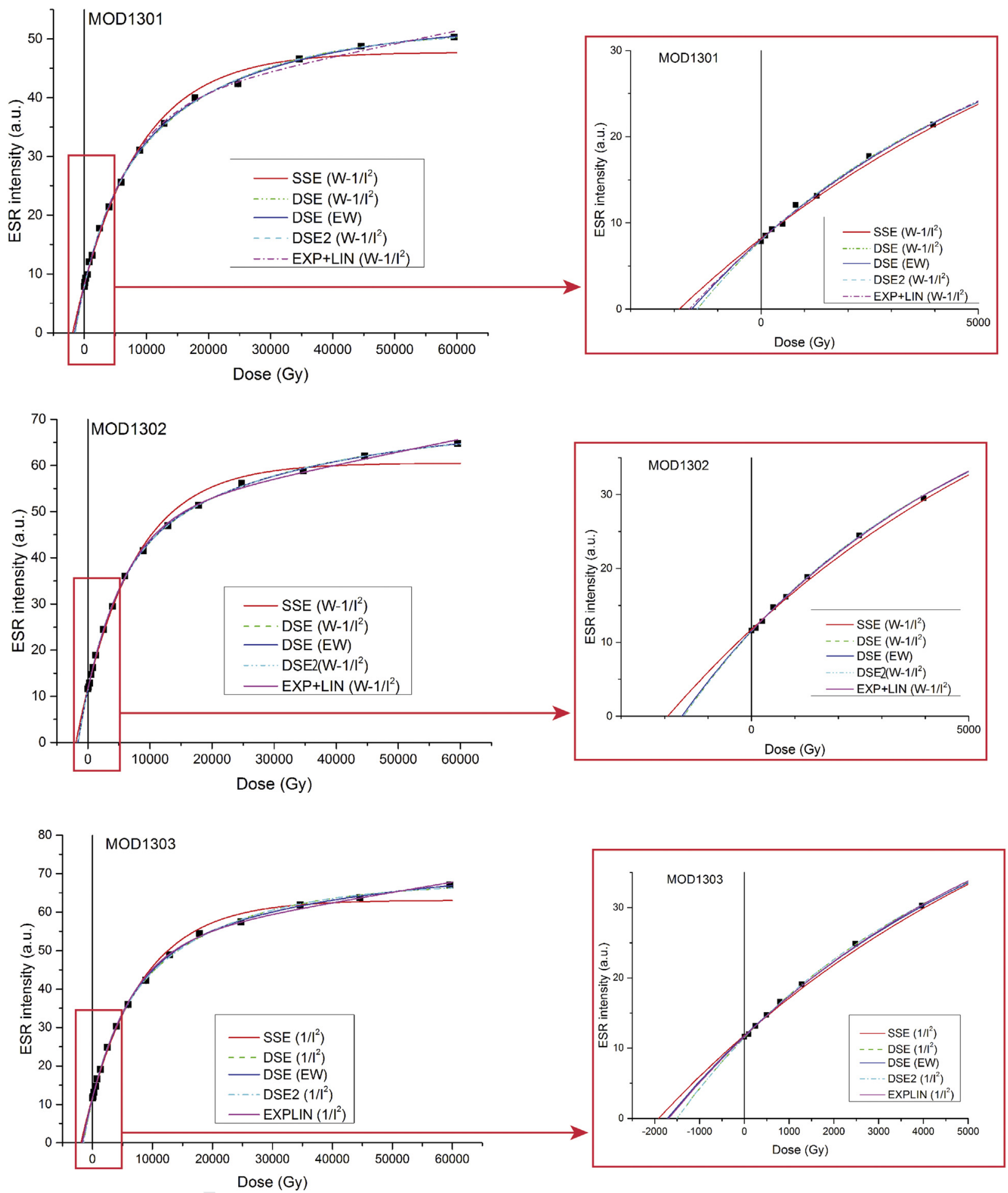

Fig. 2. Dose response curves obtained for the three samples. Numerical $D_{E}$ values are shown in Table 3 .

Fig. 3 presents the evolution $D_{E}$ values along the $D_{\max }$ for three fitting functions: DSE (equal weights and weighing by $1 / \mathrm{I}^{2}$ ), EXP + LIN and SSE. Results from the function DSE2 were not plotted since they did not provide any useful information, given the difficulty to obtain reliable fitting results when reducing the number of points. Fig. 3 shows two main patterns. On one hand, $D_{\max }$ has virtually no influence on the $D_{\mathrm{E}}$ value derived from the EXPLIN and DSE functions, whereas for the SSE, there is a clear increase of the
$D_{E}$ value along with the $D_{\max }$, which is in good agreement with previous observations (Duval et al., 2009; Chen et al., 1997). In terms of accuracy, functions EXP + LIN and SSE systematically yield overestimated $D_{E}$ values whatever the $D_{\max }$ considered. If the $\mathrm{EXP}+$ LIN function may sometimes provide $\mathrm{D}_{\mathrm{E}}$ values that consistent with the expected results, this is only because of the large error due to the limited number of data points. Nevertheless, when $D_{\max }<10 \mathrm{kGy}$, SSE provides $D_{E}$ results in agreement at 1 

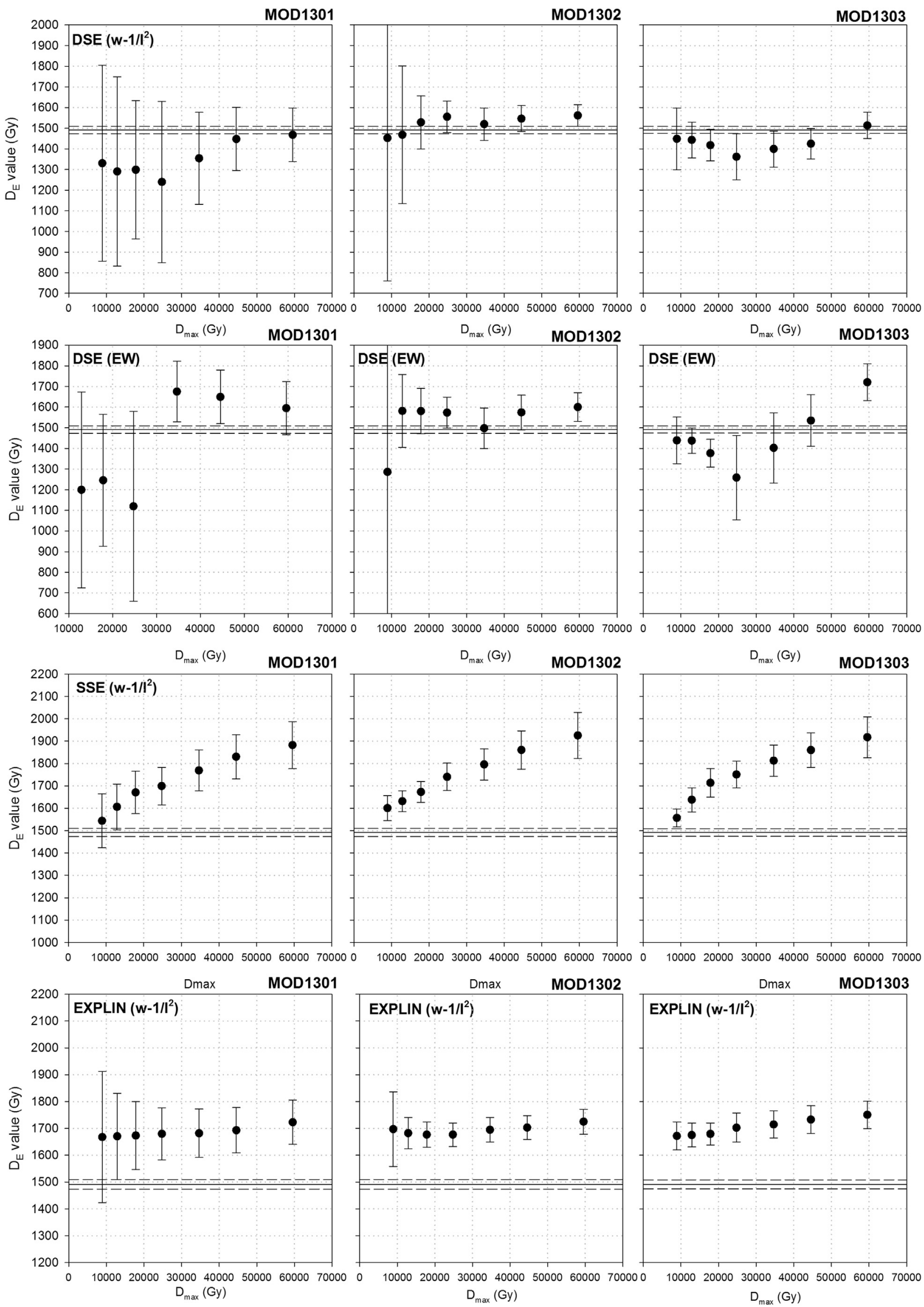

Fig. 3. Evolution of the $D_{E}$ values along with the $D_{\max }$ for the three samples based on different fitting functions: DSE, SSE and EXP + LIN. 
sigma with the expected value for 1 sample, and for the 3 samples at 2 sigmas. These results suggest that the SSE function may provide a fair estimate of the true $D_{E}$ in case $D_{\max }$ is not $>6^{*} D_{E}$. However, this empirical criterion should be used with caution since it is based on a limited number of samples. It is nevertheless worth mentioning that these observations are somewhat consistent with the simulations made by Grün and Rhodes (1992). These authors showed that in the specific case of samples with a $D_{E}$ of $0.15-0.3^{*} D_{0}$ $\left(D_{0}=\right.$ characteristic saturation dose), which correspond to the present data set, systematic overestimations occur when $D_{\max }>10^{*} D_{E}$. Their study, however, was carried out under the assumption that the behavior of the ESR signal of hydroxyapatite with the dose could be described by a SSE, which is now known to be incorrect and might actually explained part of the difference observed with the present work based on experimental data. Finally, it s worth mentioning although an adj. $\mathrm{r} 2$ value $>0.99$ is a basic requirement for an accurate dose estimation, it nevertheless do not guarantee to get a correct $\mathrm{D}_{\mathrm{E}}$ value, as observed with the SSE function. Additionally, the fact that this SSE may sometimes produce more precise $D_{E}$ values than the other functions should not be interpreted as a sign of higher reliability or accuracy, since this is simply the consequence of a limited number of parameters to fit (3).

Finally, three main observations can be made when considering the DSE function (Fig. 3):

- The $D_{E}$ error is clearly increasing when the $D_{\max }$ is decreasing. This trend may simply be explained by the fact that there are less points to be fitted in the DRC and they are further from the saturation intensity, which generates more uncertainty on the fitted parameters (see Duval et al., 2013). Consequently, the results from the present study indicate that if the DSE is apparently sometimes not suitable for the experimental ESR data set, or at least provide unreliable fitting results (e.g. Shao et al., 2014; Han et al., in press), this is most likely because of the selected $D_{\max }$ is too low.

- With equal data weighting, the $\mathrm{D}_{\mathrm{E}}$ results are in most cases consistent at 1 sigma with the expected $D_{E}$ value (14/20), and all of them are in agreement at $2 \sigma$.

- With data weighting by $1 / \mathrm{I}^{2}$, all the $\mathrm{D}_{\mathrm{E}}$ results for the 3 samples are systematically within error ( 1 sigma) with the expected $D_{E}$ value, whatever the $D_{\max }$ considered. This is therefore the best option to obtain accurate results.

\subsection{Pooling of the ESR intensities}

One of the major interests of the ESR spectroscopy is that the signal is not reset during the measurements, contrary to OSL or TL analyses for example. Consequently, a given sample may be repeatedly measured several times, and all these measurements may be included in the fitting. Pooling the ESR intensities may lead to a significant reduction of the random error on the final $D_{E}$ values (see further details in Grün and Brumby, 1994; Duval, 2012; Duval et al., 2013). In order to evaluate the impact of this increased precision on the accuracy of the final $D_{E}$, the ESR intensities obtained from the three repeated measurements were included in a single data set and a fitting was carried out with $16^{*} 3=48$ points per DRC. Numerical results are shown in Table 4 . When compared with the results obtained from the mean ESR intensities ( $=16$ points per DRC), the $D_{E}$ values are virtually the same (see Table 3 vs Table 4), but the $D_{E}$ errors are reduced by more than $50 \%$, similarly to the previous observations by Duval et al. (2013): final $D_{E}$ errors are between $1.8 \%$ and $4.6 \%$. $\mathrm{D}_{\mathrm{E}}$ values are consistent with the expected dose at $1 \sigma$ for $2 / 3$ samples, and the last one is in agreement at $2 \sigma$.
These results indicate that high precision and accuracy can be achieved when pooling the ESR intensities, provided that ESR measurements are highly repeatable.

\section{Conclusion}

The results obtained for the present study confirm those previously shown in Duval et al. (2009, 2013) and definitely demonstrate the appropriateness of the DSE fitting function for ESR dating/dosimetry of tooth samples showing $D_{\mathrm{E}}$ values $>1,000 \mathrm{~Gy}$. Consequently, they also suggest that the behavior of the radiation induced ESR signal with the dose is best described by the sum of two exponential components showing distinct saturation levels. If this general behavior that has been so far observed by the author in any tooth samples sufficiently irradiated $\left(D_{\max }>30 \mathrm{kGy}\right)$, it does not exclude, however, the possibility of some variability in the dose response of different samples within this DSE behavior. It may indeed be reasonably envisaged that each sample exhibits a specific radiation sensitivity as a result of the nature, origin and physicochemical composition of the enamel analyzed (see e.g. Porat and Schwarcz, 1994; Rink and Schwarcz, 1994). This is why further work is required to check whether these two components of the DSE may directly be correlated to the various types of $\mathrm{CO}_{2}^{-}$radicals that are mostly contributing to the signal. In addition, the reader may also have a look at the recommendations recently published by Duval et al. (2013) to optimize the fitting with a DSE function and to ensure reliable results when using Origin software. Numerical ESR data (ESR vs Dose) are provided in supplementary information so that anyone can actually test the reliability of its fitting procedure. Finally, pooling the ESR intensities derived from independent measurements for the fitting procedure may be considered as a valuable strategy for an improved precision on the $D_{E}$ values.

In contrast, the SSE function does simply not correctly describe the behavior of the radiation induced ESR signal of tooth enamel with the dose. This function systematically provides an overestimated $D_{E}$ value, which might nevertheless be sometimes in agreement with the true $D_{E}$ value under certain conditions. If this function has to be used, a special attention should be given to the maximum irradiation dose value. Our results suggest that when $D_{\max }$ does not exceed $6^{*} D_{E}$, the SSE may fairly approximate the behavior of the ESR signal with the dose. However, future investigations are required in that direction to confirm this observation and to precisely determine under which conditions of $D_{\max }$ the SSE could potentially be used. In addition, a similar study on tooth enamel samples showing lower $\mathrm{D}_{\mathrm{E}}$ values, around $200 \mathrm{~Gy}$, is ongoing, in order to check whether similar conclusions may be drawn for Middle to Late Pleistocene tooth enamel samples.

\section{Acknowledgments}

I would like to thank M. Lebon (MNHN, France) for providing the tooth samples and V. Guilarte (CENIEH, Spain) for her technical support in the early stage of the analytical procedure. The research leading to these results has received funding from the People Programme (Marie Curie Actions) of the European Union's Seventh Framework Programme (FP7/2007-2013) under REA Grant Agreement $n^{\circ}$ PIOF-GA-2013-626474. I thank the two anonymous reviewers for constructive comments.

\section{Appendix A. Supplementary data}

Supplementary data related to this article can be found at http:// dx.doi.org/10.1016/j.radmeas.2015.06.004. 


\section{References}

Apers, D., Debuyst, R., Cannière de, P., Dejehet, F., Lombard, E., 1981. Critique de la datation par résonance paramagnétique électronique (ESR) des planchers stalagmitiques de la Caune de l'Arago. Edition du CNRS. In: Lumley de, H., Labeyrie de, J. (Eds.), Datations et Analyses Isotopiques en Préhistoire: Méthodes et Limites, pp. 533-550 (preprint), Paris.

Asagoe, M., Toyoda, S., Voinchet, P., Falguères, C., Tissoux, H., Suzuki, T., Banerjee, D., 2011. ESR dating of tephra with dose recovery test for impurity centers in quartz. Quat. Int. 246 (1-2), 118-123.

Brik, A., Radchuk, V., Scherbina, O., Matyash, M., Gaver, O., 1996. Metamorphic modifications and EPR dosimetry in tooth enamel. Appl. Radiat. Isotopes 47 (11-12), 1317-1319.

Brik, A.B., Scherbina, O.I., Haskell, E.H., Sobotovich, E.V., Kalinichenko, A.M., 1997. Heating related changes in the characteristics of paramagnetic centers in tooth enamel using EPR techniques. Mineral. J. 19, 3-12.

Chen, T.-M., Yang, Q., Hu, Y.-Q., Bao, W.-B., Li, T.-Y., 1997. ESR dating of tooth enamel from yunxian homo erectus site, China. Quat. Sci. Rev. 16 (3-5), 455-458.

Curnoe, D., Grün, R., Taylor, L., Thackeray, F., 2001. Direct ESR dating of a Pliocene hominin from Swartkrans. J. Hum. Evol. 40 (5), 379-391.

Duval, M., 2012. Dose response curve of the ESR signal of the aluminum center in quartz grains extracted from sediment. Anc. TL 30 (2), 1-9.

Duval, M., Grün, R., Falguères, C., Bahain, J.J., Dolo, J.M., 2009. ESR dating of lower pleistocene fossil teeth: limits of the single saturating exponential (SSE) func tion for the equivalent dose determination. Radiat. Meas. 44 (5-6), 477-482.

Duval, M., Aubert, M., Hellstrom, J., Grün, R., 2011a. High resolution LA-ICP-MS mapping of $U$ and $T h$ isotopes in an Early Pleistocene equid tooth from Fuente Nueva-3 (Orce, Andalusia, Spain). Quat. Geochronol. 6 (5), 458-467.

Duval, M., Falguères, C., Bahain, J.-J., Grün, R., Shao, Q., Aubert, M., Hellstrom, J., Dolo, J.-M., Agusti, J., Martínez-Navarro, B., Palmqvist, P., Toro-Moyano, I., 2011b. The challenge of dating Early Pleistocene fossil teeth by the combined uranium series-electron spin resonance method: the Venta Micena palaeontological site (Orce, Spain). J. Quat. Sci. 26 (6), 603-615.

Duval, M., Falguères, C., Bahain, J.-J., 2012a. Age of the oldest hominin settlements in Spain: contribution of the combined U-series/ESR dating method applied to fossil teeth. Quat. Geochronol. 10 (0), 412-417.

Duval, M., Falguères, C., Bahain, J.-J., Grün, R., Shao, Q., Aubert, M., Dolo, J.-M., Agustí, J., Martínez-Navarro, B., Palmqvist, P., Toro-Moyano, I., 2012b. On the limits of using combined U-series/ESR method to date fossil teeth from two Early Pleistocene archaeological sites of the Orce area (Guadix-Baza basin, Spain). Quat. Res. 77 (3), 482-491.

Duval, M., Guilarte Moreno, V., Grün, R., 2013. ESR dosimetry of fossil enamel: some comments about measurement precision, long-term signal fading and dose-response curve fitting. Radiat. Prot. Dosim. 157 (4), 463-476.

Duval, M., Bahain, J.-J., Falguères, C., Garcia, J., Guilarte, V., Grün, R., Martínez, K., Moreno, D., Shao, Q., Voinchet, P., 2015. Revisiting the ESR chronology of the Early Pleistocene hominin occupation at Vallparadís (Barcelona, Spain). Quat. Int. (in press).

Grün, R., 1996. Errors in dose assessment introduced by the use of the "linear part" of a saturating dose response curve. Radiat. Meas. 26 (2), 297-302

Grün, R., 1998. Reproducibility measurements for ESR signal intensity and dose determination: high precision but doubtful accuracy. Radiat. Meas. 29 (2)
177-193.

Grün, R., Invernati, C., 1985. Uranium accumulation in teeth and its effect on ESR dating-A detailed study of a mammoth tooth. Nucl. Tracks Radiat. Meas. 10 (4-6), 869-877, 1982.

Grün, R., MacDonald, P.D., 1989. Non-linear fitting of TL/ESR dose-response curves. Appl. Radiat. Isot. 40, 1077-1080.

Grün, R., Brumby, S., 1994. The assessment of errors in past radiation doses extrapolated from ESR/TL dose-response data. Radiat. Meas. 23 (2-3), 307-315.

Grün, R., Rhodes, E.J., 1992. Simulations of saturating exponential ESR/TL dose response curves - weighting of intensity values by inverse variance. Anc. TL 10 (3), 50-56.

Hayes, R.B., Haskell, E.H., Kenner, G.H., 1998. "An assessment of the LevenbergMarquardt fitting algorithm on saturating exponential data sets.". Anc. TL 16 (2), 57-62.

Han, F., Bahain, J.-J., Boëda, É., Hou, Y., Huang, W., Falguères, C., Rasse, M., Wei, G., Garcia, T., Shao, Q., Yin, G., 2012. Preliminary results of combined ESR/U-series dating of fossil teeth from Longgupo cave, China. Quat. Geochronol. 10 (0), 436-442.

Han, F., Bahain, J.-J., Liu, C., Yin, G., 2015. Testing mathematical uranium migration models in combined ESR/U-series dating of fossil teeth from open-air sites. Quat. Geochronol. http://dx.doi.org/10.1016/j.quageo.2015.05.009 (in press).

Hoffmann, D., Mangini, A., 2002. Comparative studies on the $\mathrm{CO}_{2}^{-}$signal in tooth enamel and Carbonates. Radiat. Prot. Dosim. 101 (1-4), 359-362.

Joannes-Boyau, R., Grün, R., 2011. A comprehensive model for CO2- radicals in fossil tooth enamel: Implications for ESR dating. Quat. Geochronol. 6 (1), 82-97. Kohn, M.J., Schoeninger, M.J., Barker, W.W., 1999. Altered states: effects of diagenesis on fossil tooth chemistry. Geochim. Cosmochim. Acta 63 (18), 2737-2747.

Murray, A.S., Wintle, A.G., 2003. The single aliquot regenerative dose protocol: potential for improvements in reliability. Radiat. Meas. 37 (4-5), 377-381.

Nilsson, J., Lund, E., Lund, A., 2001. The effects of UV-irradiation on the ESRdosimetry of tooth enamel. Appl. Radiat. Isotopes 54 (1), 131-139.

Porat, N., Schwartz, H.P., 1994. ESR dating of tooth enamel: a universal growth curve. In: Corrucini, R., Ciochon, R. (Eds.), Inregrarive Parhs ro rhe Pasr: Paleoanrhropological Aabmces in Honor of F. C. Howell. Prentice-Hall, Englewood Cliffs, pp. 521-530.

Rink, J.W., Schwarcz, H.P., 1994. Dose response of ESR signals in tooth enamel. Radiat. Meas. 23 (2-3), 481-484.

Rink, W.J., Schwarcz, H.P., 1995. Tests for diagenesis in tooth enamel: ESR dating signals and carbonate contents. J. Archaeol. Sci. 22 (2), 251-255.

Schwarcz, H.P., Grün, R., Tobias, P.V., 1994. ESR dating studies of the australopithecine site of Sterkfontein, South Africa. J. Hum. Evol. 26 (3), 175-181.

Shao, Q., Wang, W., Deng, C., Voinchet, P., Lin, M., Zazzo, A., Douville, E., Dolo, J.-M., Falguères, C., Bahain, J.-J., 2014. ESR, U-series and paleomagnetic dating of Gigantopithecus fauna from Chuifeng Cave, Guangxi, southern China. Quat. Res. 82 (1), 270-280.

Tiemei, C., Qi, C., Quan, Y., Yanqiu, H., 2001. The problems in ESR dating of tooth enamel of Early Pleistocene and the age of Longgupo hominid, Wushan, China. Quat. Sci. Rev. 20 (5-9), 1041-1045.

Vanhaelewyn, G., Callens, F., Grün, R., 2000. EPR spectrum deconvolution and dose assessment of fossil tooth enamel using maximum likelihood common factor analysis. Appl. Radiat. Isotopes 52 (5), 1317-1326. 